\title{
Avaliação da Gestão do Programa Nacional de Alimentação Escolar sob a ótica do fomento da agricultura familiar
}

\section{Management Evaluation of the National School Feeding Program from the perspective of fostering family farming}

\author{
Priscila Porrua' (D), Daniele Cristina da Silva Kazama', Cristine Garcia Gabriel2,3, \\ Gabriele Rockenbach², Maria Cristina Marino Calvo ${ }^{4}$, Patrícia de Oliveira Machado ${ }^{4}$, \\ Janaina das Neves ${ }^{2,3}$, Ruan Weiss ${ }^{5}$
}

'Programa de Pós-graduação em Agroecossistemas, Universidade Federal de Santa Catarina (UFSC) - Florianópolis (SC), Brasil ${ }^{2}$ Departamento de Nutrição, Universidade Federal de Santa Catarina (UFSC) - Florianópolis (SC), Brasil

${ }^{3}$ Programa de Pós-graduação em Nutrição, Universidade Federal de Santa Catarina (UFSC) - Florianópolis (SC), Brasil. ${ }^{4}$ Programa de Pós-graduação em Saúde Coletiva, Universidade Federal de Santa Catarina (UFSC) - Florianópolis (SC), Brasil.

${ }^{5}$ Curso de Nutrição, Universidade Federal de Santa Catarina (UFSC) - Florianópolis (SC), Brasil.

Como citar: Porrua P, Kazama DCS, Gabriel CG, Rockenbach G, Calvo MCM, Machado PO, et al. Avaliação da Gestão do Programa Nacional de Alimentação Escolar sob a ótica do fomento da agricultura familiar. Cad Saúde Colet, 2020;28(3):426-437. https://doi.org/10.1590/1414-462X202028030361

\section{Resumo}

Introdução: A compra institucional para a alimentação escolar é uma oportunidade de incentivo a novas cadeias de abastecimento, sobretudo em nível local. Objetivo: Avaliar a gestão municipal do Programa Nacional de Alimentação Escolar (PNAE) relacionada ao fomento e aquisição de alimentos da agricultura familiar. Método: Foi realizado levantamento bibliográfico de estudos avaliativos no âmbito do PNAE para seleção dos indicadores e medidas pertinentes ao objeto e desenvolvido um modelo avaliativo. O modelo avaliativo foi pactuado após submissão a oficinas de consenso. Foi elaborado um questionário e enviado por meio eletrônico a 18 municípios de Santa Catarina. A amostra foi definida de forma intencional, e os municípios selecionados por meio de sorteio. Para avaliar as ações da gestão municipal do PNAE destinadas ao fomento e aquisição de alimentos da agricultura familiar, foram utilizados 5 indicadores, 9 medidas e suas respectivas questões. Resultados: A avaliação dos municípios foi predominantemente "regular" (88\%; n=14). Conclusão: A avaliação apontou fragilidades da gestão municipal em relação ao desconhecimento da legislação e não aplicabilidade dos critérios previstos em normativa, especialmente quanto ao Planejamento de Cardápios, realização de Chamada Pública e seleção dos Projetos de Venda. Palavras-chave: alimentação escolar; avaliação; agricultura familiar.

\footnotetext{
Abstract

Background: The institutional purchase for school feeding is an opportunity to encourage new supply chains, especially at the local level. Objective: To evaluate the municipal management of the National School Feeding Program (PNAE) related to the promotion and acquisition of food from family agriculture. Method: A bibliographic survey of evaluative studies was carried out within the scope of the PNAE to select indicators and measures pertinent to the object and develop an evaluation model. The evaluative model was agreed after submission to consensus workshops. A questionnaire was developed and sent electronically to 18 municipalities of Santa Catarina. The sample was intentionally defined and the municipalities selected by lot. In order to evaluate the actions of municipal management of the PNAE for the promotion and acquisition of food from family agriculture, five indicators, 9 measures and their

Trabalho foi desenvolvido como produto do Centro Colaborador em Alimentação e Nutrição do Escolar de Santa Catarina (CECANE/SC), em parceria com o Fundo Nacional de Desenvolvimento da Educação (FNDE) - Florianópolis (SC), Brasil. Correspondência: Priscila Porrua. E-mail: pporrua@gmail.com

Fonte de financiamento: Fundo Nacional de Desenvolvimento da Educação (FNDE), Centro Colaborador em Alimentação e Nutrição do Escolar de Santa Catarina, termo de execução n 4767/2016.

Conflito de interesses: nada a declarar.

Recebido em: Mar. 09, 2018. Aprovado em: Set. 16, 2019
}

ISSN 2358-291X (Online) 
respective issues were used. Results: The evaluation of municipalities was predominantly "regular" $(88 \%, n=14)$. Conclusion: The evaluation pointed to fragilities of municipal management in relation to the lack of knowledge of the legislation and non-applicability of the criteria set forth in regulations, especially regarding menu planning, public call and selection of sales projects.

Keywords: school feeding; evaluation; family farming.

\section{INTRODUÇÃO}

A Alimentação Escolar (AE), até a década de 1990, seguiu predominantemente a lógica dos mercados autorregulados cujo modelo de produção de alimentos é pautado em cadeias longas de abastecimento, na agricultura intensiva, mecanizada, com elevada utilização de produtos químicos, crescente processamento dos alimentos e padronização de hábitos alimentares'. Até o ano de 1993, o planejamento dos cardápios, aquisição e distribuição de alimentos no território nacional eram de responsabilidade da gestão federal. Em 1994, com a promulgação da Lei n 8.913/1994, os recursos do Programa Nacional de Alimentação Escolar (PNAE) passaram a ser descentralizados aos municípios e estados. Já em 1998, a Medida no 1.784 retirou a necessidade de celebração de convênios como requisito para o recebimento de recursos e instituiu a transferência automática, potencializando a efetivação da aquisição e acesso a alimentos locais em quantidade e qualidade adequadas ${ }^{2}$.

Em 2001, a Medida Provisória $n^{\circ} 2.178$ instituiu que, dentre os recursos federais transferidos à conta do PNAE no município ou estado, obrigatoriamente, $70 \%$ deviam ser utilizados para aquisição de alimentos básicos que respeitassem os hábitos alimentares regionais e locais, vocação agrícola do município, com vistas ao fomento e desenvolvimento da economia local ${ }^{2}$.

Neste contexto, e diante de questionamentos quanto ao modelo de desenvolvimento com efeitos controversos à sociedade e agroecossistemas, a $\mathrm{AE}$ começou a ser vista como oportunidade para novas cadeias de abastecimento, em que a combinação do incentivo à produção agrícola local com a qualificação dos programas de AE seriam meios de promover a Segurança Alimentar e Nutricional (SAN) ${ }^{1,3}$.

Assim, o incentivo ao cumprimento das normativas voltadas à execução do PNAE, bem como obrigatoriedade instituída pela Lei no 11.947/2009 em relação à destinação de no mínimo $30 \%$ dos recursos para aquisição de alimentos provenientes da Agricultura Familiar (AF), têm papel imprescindível no alcance das diretrizes do Programa relacionadas ao fomento de novas cadeias de abastecimento por meio das compras institucionais em nível local ${ }^{4-6}$.

Diante da estreita relação entre o desempenho da gestão e o alcance dos objetivos do Programa, o presente trabalho propõe avaliar a gestão municipal do PNAE quanto a indicadores relacionados ao fomento e aquisição de alimentos da Agricultura Familiar.

\section{MÉTODO}

Este estudo integra uma pesquisa de avaliação normativa de maior extensão, intitulada Avaliação da gestão do PNAE em municípios do estado de Santa Catarina, aprovada pelo Comitê de Ética e Pesquisa com Seres Humanos (CEPSH) da Universidade Federal de Santa Catarina (parecer no 1377341). A avaliação normativa consiste na emissão de juízo de valor sobre uma intervenção, comparando-se os critérios previstos nas normativas com o que é executado ${ }^{7}$. Refere-se à comparação entre recursos empregados e a estrutura, os serviços ou os processos e os resultados obtidos.

Esta pesquisa foi desenvolvida pelo Centro Colaborador em Alimentação e Nutrição do Escolar de Santa Catarina (CECANE/SC), em parceria com o Fundo Nacional de Desenvolvimento da Educação (FNDE). Sua execução se deu em duas etapas: desenvolvimento de um modelo avaliativo e coleta de dados com a gestão municipal, conduzidas entre 2015 e 2016.

Na primeira etapa, houve o desenvolvimento do modelo avaliativo com o levantamento bibliográfico de estudos avaliativos no âmbito do PNAE, para a seleção de indicadores e medidas pertinentes ao objeto. O modelo avaliativo foi submetido a oficinas de consenso com especialistas nas áreas de nutrição, alimentação escolar e avaliação de políticas públicas, no formato de comitês tradicionais ${ }^{8}$.

Na segunda etapa houve a aplicação do modelo avaliativo, por meio de um questionário eletrônico. A amostra de municípios foi definida de forma intencional, contemplando um 
município de cada estrato populacional por mesorregião de Santa Catarina, no total de 18 municípios. Para selecionar os participantes, foi realizado sorteio entre os municípios existentes por porte (pequeno, médio e grande) e mesorregião (Grande Florianópolis, Norte, Oeste, Serrana, Sul, Vale do Itajaí), utilizando o programa Excel ${ }^{\circledast}$ (Microsoft Excel, 2007). O número de habitantes por município foi obtido por meio das estimativas de porte municipal realizadas pelo Instituto Brasileiro de Geografia e Estatística (IBGE) de acordo com o Censo Populacional ${ }^{9}$. Na Tabela 1 estão descritos os municípios com o número de habitantes e número de escolares assistidos pelo PNAE.

Tabela 1. Municípios catarinenses que participaram da avaliação com os respectivos números de habitantes e número de unidades escolares (UEs). Santa Catarina - SC, 2016

\begin{tabular}{|c|c|c|c|}
\hline Mesorregião & Município & Número de habitantes ${ }^{a}$ & $\begin{array}{l}\text { Número de escolares } \\
\text { da educação básicab }\end{array}$ \\
\hline \multicolumn{4}{|c|}{ PEQUENO PORTE (<20.000 habitantes) } \\
\hline Vale do Itajaí & M1 & 11.380 & 1.218 \\
\hline Sul & M2 & 11.231 & 466 \\
\hline Norte & M3 & 7.899 & 554 \\
\hline Grande Florianópolis & M4 & 2.849 & 214 \\
\hline Serrana & M5 & 2.630 & 337 \\
\hline Oeste & M6 & 1.943 & 48 \\
\hline \multicolumn{4}{|c|}{ MÉDIO PORTE (de 20.001 a 100.000 habitantes) } \\
\hline Norte & M7 & 48.606 & 4.590 \\
\hline Serrana & M8 & 26.247 & 1.184 \\
\hline Oeste & M9 & 24.345 & 2.133 \\
\hline \multicolumn{4}{|c|}{ GRANDE PORTE (> 100.000 habitantes) } \\
\hline Norte & M10 & 562.151 & 59.310 \\
\hline Vale do Itajaí & M11 & 338.876 & 32.211 \\
\hline Grande Florianópolis & M12 & 232.309 & 10.332 \\
\hline Oeste & M13 & 205.795 & 18.052 \\
\hline Sul & M14 & 206.918 & 14.682 \\
\hline Serrana & M15 & 158.732 & 14.589 \\
\hline Grande Florianópolis & M16 & 157.833 & 9.875 \\
\hline
\end{tabular}

Fonte: alBGE Cidades ${ }^{9}$; IInstituto Nacional de Estudos e Pesquisas Educacionais Anísio Teixeira (INEP) ${ }^{10}$

Para a coleta de dados, foi utilizado um formulário eletrônico desenvolvido por meio do software Google docs ${ }^{\circledast}$ (Google Inc., 2016). Foram enviados e-mails com textos padronizados aos gestores municipais (secretário (a) de educação) com cópia para os nutricionistas Responsáveis Técnicos (RT) e que continham, além do link para acesso ao questionário, quatro anexos: 1) questionário em $p d f$ para consulta; 2) glossário com termos-chave; 3) carta de apresentação com informações pertinentes à pesquisa e 4) termo de consentimento livre e esclarecido. Os municípios também foram contatados por telefone para confirmação do recebimento do e-mail e esclarecimentos.

Para avaliar as ações da gestão municipal do PNAE destinadas ao fomento e aquisição de alimentos da AF, foram utilizados 5 indicadores, 9 medidas (Quadro 1) e suas respectivas 22 questões ${ }^{11}$. 


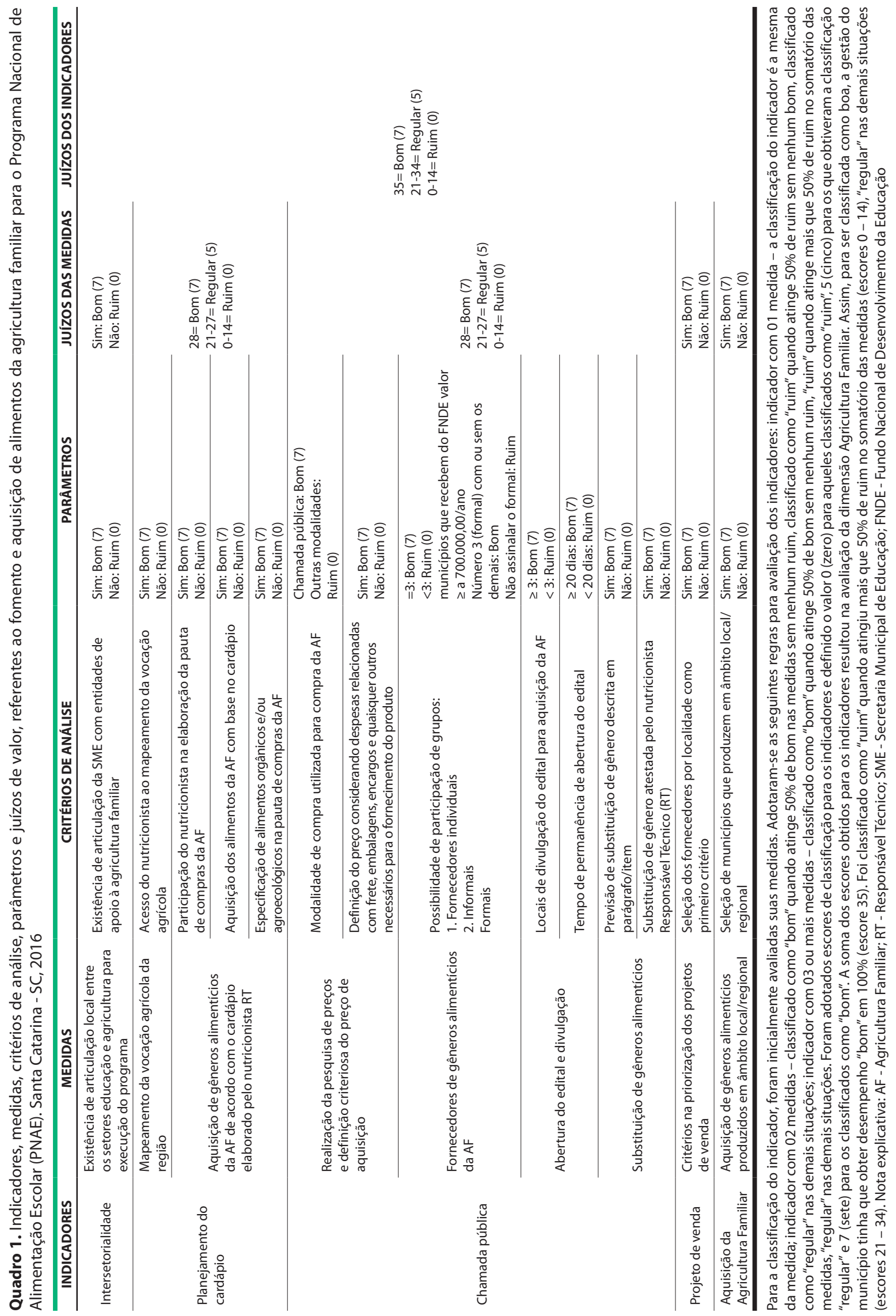


O primeiro indicador, Articulação entre atores, foi avaliado por meio de uma medida: Existência de articulação local entre os setores educação e agricultura para execução do programa, no ano de 2015. Este indicador foi proposto considerando o que está previsto nas normativas do PNAE e SAN $\mathrm{N}^{6,12-14}$.

O indicador Planejamento do cardápio foi avaliado por meio de duas medidas, referentes ao ano de 2015: 1. Mapeamento da vocação agrícola da região - que avaliou o acesso do nutricionista RT a esse mapeamento, considerando inclusive a produção orgânica e agroecológica; 2 . Aquisição de gêneros alimentícios da AF de acordo com o cardápio elaborado pelo nutricionista RT - medida que avaliou a aquisição de alimentos da AF e a especificação de alimentos orgânicos e/ou agroecológicos na pauta de compras.

O terceiro indicador, Chamada pública, foi avaliado por meio de quatro medidas: 1. Realização da pesquisa de preços e definição criteriosa do preço de aquisição - avaliou a modalidade de compra e critérios considerados na pesquisa de preço. 2 . Fornecedores de gêneros alimentícios da AF - avaliou se foram contemplados fornecedores individuais, informais e formais; 3 . Abertura do edital e divulgação - avaliou a diversidade de locais onde o edital foi divulgado e o tempo que permaneceu aberto; 4. Substituição de gêneros alimentícios - avaliou a previsão e aprovação das substituições.

O quarto indicador, Projeto de venda, foi avaliado por meio de uma medida: Critérios na priorização dos projetos de venda, em 2015 - que considerou a localidade como critério prioritário, conforme a Resolução CD/FNDE no 04/2015 ${ }^{13}$.

O quinto e último indicador, Aquisição da $A F$, foi avaliado por meio de uma medida: Aquisição de gêneros alimentícios produzidos em âmbito local/regional, para alimentação escolar - que avaliou se há produção local, se foram contemplados os produtores e se foram adquiridos alimentos de outras regiões, em 2015.

O juízo de valor do referido modelo avaliativo foi estabelecido com base no estudo de Gabriel ${ }^{11}$. Todos os indicadores foram considerados com o mesmo grau de importância e classificados como "bom", "regular" ou "ruim" segundo os parâmetros definidos na matriz (Quadro 1).

\section{RESULTADOSE DISCUSSÃO}

Obteve-se adesão de 16 municípios catarinenses, sendo que 2 alegaram não ter interesse em participar da pesquisa. O número de habitantes variou de 1.943 (pequeno porte) a 562.151 (grande porte), e dentre o número de escolares houve variação de 48 a 59.310 .

Os municípios avaliados segundo os indicadores investigados (Quadro 2) apresentaram predomínio do desempenho "regular" $(88 \% ; n=14)$ para a avaliação da gestão municipal. Observou-se maior percentual de municípios com desempenho "bom" nos indicadores Articulação entre atores (94\%; $\mathrm{n}=15)$ e Aquisição da $A F(81 \% ; n=13)$. Dois municípios (12\%) apresentaram desempenho "ruim" na classificação final.

Quadro 2. Resultados da avaliação da gestão municipal do Programa Nacional de Alimentação Escolar (PNAE) segundo indicadores da agricultura familiar, por município. Santa Catarina - SC, 2016

\begin{tabular}{ccccccc}
\hline & \multicolumn{7}{c}{ Dimensão: Agricultura Familiar } \\
\cline { 1 - 4 } Indicadores & $\begin{array}{c}\text { Articulação } \\
\text { entre atores }\end{array}$ & $\begin{array}{c}\text { Planejamento } \\
\text { de cardápio }\end{array}$ & $\begin{array}{c}\text { Chamada } \\
\text { pública }\end{array}$ & $\begin{array}{c}\text { Projeto } \\
\text { de } \\
\text { venda }\end{array}$ & $\begin{array}{c}\text { Aquisição } \\
\text { da } \\
\text { Agricultura } \\
\text { Familiar }\end{array}$ & $\begin{array}{c}\text { Classificação } \\
\text { dos } \\
\text { municípios }\end{array}$ \\
\cline { 1 - 7 } Municípios & Bom & Regular & Regular & Ruim & Bom & Regular \\
\hline M1 & Bom & Regular & Regular & Ruim & Bom & Regular \\
\hline M3 & Bom & Regular & Ruim & Ruim & Bom & Regular \\
\hline M4 & Bom & Regular & Regular & Bom & Bom & Regular \\
\hline M5 & Bom & Regular & Ruim & Ruim & Ruim & Ruim \\
\hline M6 & Bom & Regular & Regular & Bom & Bom & Regular \\
\hline M7 & Bom & Regular & Ruim & Bom & Bom & Regular \\
\hline
\end{tabular}


Quadro 2. Continuação...

\begin{tabular}{|c|c|c|c|c|c|c|}
\hline \multicolumn{7}{|c|}{ Dimensão: Agricultura Familiar } \\
\hline Indicadores & $\begin{array}{l}\text { Articulação } \\
\text { entre atores }\end{array}$ & $\begin{array}{l}\text { Planejamento } \\
\text { de cardápio }\end{array}$ & $\begin{array}{l}\text { Chamada } \\
\text { pública }\end{array}$ & $\begin{array}{c}\text { Projeto } \\
\text { de } \\
\text { venda }\end{array}$ & $\begin{array}{l}\text { Aquisição } \\
\text { da } \\
\text { Agricultura } \\
\text { Familiar }\end{array}$ & $\begin{array}{c}\text { Classificação } \\
\text { dos } \\
\text { municípios }\end{array}$ \\
\hline Municípios & \multicolumn{6}{|c|}{ Juízos de valor } \\
\hline M8 & Bom & Regular & Regular & Ruim & Bom & Regular \\
\hline M9 & Bom & Regular & Regular & Ruim & Bom & Regular \\
\hline M10 & Bom & Regular & Regular & Ruim & Ruim & Regular \\
\hline M11 & Bom & Regular & Regular & Bom & Bom & Regular \\
\hline M12 & Bom & Regular & Regular & Bom & Bom & Regular \\
\hline M13 & Bom & Bom & Regular & Bom & Bom & Regular \\
\hline M14 & Bom & Regular & Regular & Ruim & Bom & Regular \\
\hline M15 & Bom & Regular & Bom & Bom & Bom & Regular \\
\hline M16 & Ruim & Regular & Ruim & Ruim & Ruim & Ruim \\
\hline
\end{tabular}

\section{Articulação entre atores}

A Articulação entre atores foi classificada como"bom"em 94\% ( $n=15)$ dos municípios que afirmaram realizar articulação entre a Secretaria Municipal de Educação (SME) com alguma entidade de apoio à AF. As entidades de articulação citadas foram identificadas: Secretaria de Agricultura; Sindicato dos Trabalhadores Rurais/Sindicato Rural; Entidades de Assistência Técnica Rural (ATER) e Organizações Não Governamentais (ONGs). Somente um município afirmou não realizar tal articulação.

Dentre as entidades assinaladas, o apoio da ATER foi a mais citada $(n=11)$ com articulação com a SME, seguida da Secretaria de Agricultura $(n=8)$, ONGs $(n=7)$, Sindicato Rural $(n=5)$.

Quanto à articulação com entidades de ATER, é válido ressaltar que em Santa Catarina (SC), em 1991, foi criada a Empresa de Pesquisa Agropecuária e Extensão Rural (EPAGRI). Ainda que a EPAGRI não possua assistência técnica direcionada à inclusão da AF no mercado institucional, especificamente para o PNAE, o diálogo com as SME possibilita o fortalecimento da AF. A EPAGRI tem importante abrangência, presta mais de 350 mil assistências anuais a agricultores familiares, pescadores artesanais e comunidades indígenas e quilombolas, além de promover ações dirigidas a mulheres e jovens rurais ${ }^{15}$, em SC.

Outro destaque em relação à assistência técnica são os Centros Colaboradores em Alimentação e Nutrição do Escolar (CECANE), unidades de referência criadas em parcerias entre as universidades federais e o FNDE, com o objetivo de desenvolver ações para a qualificação do PNAE ${ }^{16}$. No caso de SC, o CECANE atua desde 2008 junto aos 295 municípios do estado e é considerado um importante parceiro no fomento da aquisição de alimentos da AF para o PNAE.

A articulação entre as SME e tais entidades de apoio à AF, como a EPAGRI, entre outros, se faz necessária para esclarecimentos sobre os trâmites de comercialização, servindo de apoio no processo de inclusão de produtores no mercado institucional ${ }^{3,17}$. Para além dos setores educação e agricultura, é reconhecida a importância da articulação dos programas de AE com outros setores, como a saúde. Avaliação sobre a gestão municipal do PNAE nas três capitais do Sul do Brasil verificou que as ações entre os setores da educação e saúde influenciaram positivamente no monitoramento alimentar e nutricional dos escolares ${ }^{18}$. Nesta perspectiva, estudo com municípios de SC destacou a intersetorialidade e a relação entre os campos da educação, saúde, agricultura, assistência como importantes estratégias de superação dos desafios às gestões municipais para implementação das normativas do PNAE $^{19}$.

Neste contexto, a gestão dos municípios analisados está de acordo com a Resolução CD/FNDE n²6/2013 ${ }^{12}$, que prevê que a efetivação da intersetorialidade no PNAE se realize por meio de ações conjuntas entre agricultura, saúde e outros ${ }^{20}$. 


\section{Planejamento do cardápio}

No planejamento dos cardápios foi observado que $94 \%(n=15)$ dos municípios apresentaram avaliação "regular". Este desempenho está relacionado principalmente à dificuldade de acesso ao mapeamento da vocação agrícola da região, pois $75 \%(n=12)$ dos municípios relataram não ter acesso.

O planejamento do cardápio, a ser realizado pelo nutricionista RT considerando a vocação agrícola da região, é o primeiro passo para a inclusão dos produtores locais no PNAE $E^{6,12,21}$. Para isso, a Resolução no 465/2010, que dispõe sobre as atribuições do nutricionista no PNAE, estabelece que este RT deve "[...] interagir com os agricultores e empreendedores familiares rurais, e suas organizações, de forma a conhecer a produção local, inserindo esses produtos na $A E[\ldots]^{\prime \prime 22}$.

A dificuldade de realização e acesso ao mapeamento da vocação agrícola da região pelo nutricionista RT pode estar relacionada à não articulação entre este profissional com membros do Conselho de Alimentação Escolar (CAE), gestão, agricultores e representantes de entidades de apoio à $A^{21,23}$. Isso pode ocorrer devido ao acúmulo de funções deste profissional nos casos em que há um quadro inadequado de nutricionistas por número de escolares atendidos, mas principalmente à formação desses profissionais ${ }^{24}$ por instituições de ensino superior que condicionam o olhar para a saúde em seu conceito reducionista, de forma fragmentada e descontextualizada.

Neste sentido, sugere-se que a gestão proporcione atividades de formação permanente aos nutricionistas $\mathrm{RT}$ e membros do CAE, para fomentar a articulação entre estes e os produtores da AF e demais agentes do Programa, com vistas a estabelecer uma relação de empoderamento e reciprocidade entre os agentes, contemplando as expectativas e demandas específicas de cada localidade ${ }^{21,23,25}$.

Além disso, recomenda-se a elaboração e atualização do mapeamento agrícola e disponibilização em domínio público, contendo informações como quantidade de produção, época de colheita e diversidade de produtos. Esta ação poderia ser realizada em parceria entre Secretaria de Agricultura, Educação, CAE, Entidades de apoio à AF, e auxiliaria nutricionistas de municípios próximos, fortalecendo o diálogo entre os nutricionistas e a AF, facilitando o planejamento dos cardápios com a inclusão de alimentos da $\mathrm{AF}$, respeito à sociobiodiversidade e sazonalidade ${ }^{21}$.

Após o planejamento do cardápio, com base na vocação agrícola da região, o nutricionista RT deve elaborar a pauta para aquisição de alimentos, que será entregue ao setor de compras, que por sua vez realiza a divulgação no edital de chamada pública' ${ }^{12,13}$.

Quanto à inclusão de alimentos orgânicos/agroecológicos provenientes da $A F$, foi observado que metade da amostra $(n=8)$ afirmou não haver especificação destes alimentos na pauta de compras.

A Resolução CD/FNDE n²6/2013 estabelece a prioridade dos alimentos orgânicos e/ou agroecolológicos em relação aos convencionais ${ }^{12}$. Assim, a inserção destes alimentos na pauta de compras e publicação destes no edital, com a discriminação de que sejam orgânicos e/ou agroecológicos, faz com que se efetive a prioridade prevista em normativa.

O mapeamento da produção de alimentos e o planejamento do cardápio com uma criteriosa elaboração da pauta de compras, com a discriminação dos orgânicos e/ou agroecológicos, são considerados fatores imprescindíveis para potencializar a inclusão desses no cardápio, auxiliando os agricultores no planejamento da produção, promovendo o respeito à sazonalidade e adequação entre a oferta e demanda ${ }^{21,23,26,27}$.

\section{Chamada pública}

Neste indicador, 69\% ( $n=11)$ dos municípios obtiveram desempenho "regular", e apenas um obteve "bom" desempenho. Em relação à modalidade de compra, foi observado que todos os municípios $(n=16)$ realizaram chamada pública para aquisição de alimentos da AF. A Resolução CD/FNDE no 26/2013 12 sugere que a compra de alimentos da AF seja por meio da dispensa do procedimento licitatório, utilizando a chamada pública. Esta iniciativa está 
relacionada à aquisição na perspectiva de SAN, considerando a sustentabilidade, inclusão e fortalecimento da $\mathrm{AF}$, incentivo ao desenvolvimento local e demais valores em detrimento do menor preço e concorrência' ${ }^{1}$. Neste sentido, torna-se imprescindível a qualificação da gestão e principalmente do setor de compras das Entidades Executoras (EEx.), para esclarecimentos sobre as modalidades de compra e contextualização da importância da chamada pública para aquisição de alimentos da AF.

Foi observado que $69 \%(n=11)$ dos municípios afirmaram priorizar a localidade na realização da pesquisa de preço, atendendo a sequência de prioridade aos três mercados locais, regionais, estaduais ou nacionais. Porém, quanto ao preço de aquisição, foi observado que $25 \%(n=4)$ dos municípios não consideraram as despesas relacionadas com frete, embalagens, encargos e quaisquer outros necessários para o fornecimento dos produtos. Estes municípios estão em inconformidade com os requisitos estabelecidos na Resolução CD/FNDE n 04/2015, que determina que o preço de aquisição seja o preço médio pesquisado por, no mínimo, três mercados em âmbito local, priorizando a feira do produtor da AF, quando houver, acrescido dos insumos exigidos no edital de chamada pública, tais como despesas com frete, embalagens, encargos e quaisquer outros necessários para o fornecimento do produto ${ }^{13}$.

A formação equivocada do preço de aquisição é um dos entraves na inclusão dos agricultores familiares, pois nestes casos o valor pago pelos alimentos não cobre as despesas com logística, embalagem, custos administrativos de cooperativas e associações, desestimulando e excluindo muitos agricultores'.

Em relação aos critérios de seleção dos fornecedores, foi observado que 44\% ( $n=7)$ dos municípios não os aplicaram corretamente. Além disso, destaca-se que $31 \%(n=5)$ dos municípios não tiveram ampla divulgação da abertura do edital para aquisição da $A F$, em inconformidade com a Resolução CD/FNDE n $04 / 2015^{13}$. Também houve inconformidade em relação ao tempo de abertura do edital para aquisição de alimentos da $A F$, onde $31 \%(n=5)$ dos municípios afirmaram deixar o edital aberto por menos de 20 dias, enquanto a normativa prevê que "[...] os editais das chamadas públicas deverão permanecer abertos para recebimento dos projetos de venda por um período mínimo de 20 dias ${ }^{13^{\prime \prime}}$.

Com relação às substituições dos gêneros alimentícios provenientes da $A F$, foi verificado que $81 \%$ ( $n=13$ ) dos municípios afirmaram não haver no edital um parágrafo prevendo possíveis substituições destes. A Resolução CD/FNDE n 04/2015 determina que "[...] os alimentos a serem entregues ao contratante serão os definidos na chamada pública de compra, podendo ser substituídos quando houver necessidade, desde que os produtos substitutos constem na mesma chamada pública e sejam correlatos nutricionalmente e que a substituição seja atestada pelo RT, que poderá contar com o respaldo do $\mathrm{CAE}^{13 \prime}$.

Desta forma, torna-se fundamental a articulação entre o nutricionista e os produtores de alimentos para definição das possibilidades de substituição de alimentos, descrevendo-os na chamada pública.

\section{Projeto de venda}

Foi observado que $56 \%(n=9)$ dos municípios não priorizaram os grupos de projetos de venda de fornecedores locais sobre os demais grupos. Após o recebimento dos projetos de venda, deve haver a organização dos projetos em quatro grupos, priorizando os projetos de venda locais (pertencentes ao território do município da EEx); do território rural; estaduais e nacionais, nesta ordem ${ }^{13}$.

Alguns estudos têm evidenciado justificativas para esta exclusão de projetos de venda locais. Estas estão relacionadas aos indicadores anteriores no presente estudo, como a falta de assistência técnica e apoio para esclarecimentos ao agricultor que pretende se inserir; deficiente articulação entre o nutricionista, agricultor e demais agentes do Programa; ausência de mapeamento da produção local; planejamento de cardápio descontextualizado 1,17,21,23-27. Estas características têm como consequência reclamações por parte da gestão em relação à falta de projetos locais, quantidades insuficientes de oferta alimentos, variedade insuficiente de alimentos, falta de documentação do agricultor ${ }^{5,28}$ e culminando na exclusão de agricultores 
locais e menos consolidados. Além disso, a gestão desqualificada e desconhecimento dos critérios de prioridade estabelecidos em normativa também acarretam prejuízos e exclusão de produtores locais ${ }^{25}$.

Ainda em relação aos critérios de priorização, é válido ressaltar que, após organização dos grupos de projetos (locais, território rural, estaduais e nacionais), há uma segunda etapa na seleção dos projetos de venda, cuja prioridade é: assentamentos de reforma agrária, comunidades tradicionais indígenas, comunidades quilombolas, não havendo prioridade entre eles; produtores de alimentos orgânicos/agroecológicos certificados; grupos formais (DAP jurídica); grupos informais (agricultores familiares organizados em grupo, utilizando suas DAP físicas); fornecedores individuais (DAP física) ${ }^{13}$.

Como as etapas de seleção dos projetos de venda devem ser realizadas em sessão pública e registradas em ata, para minimizar a ocorrência de inconformidades, recomenda-se a participação de um representante do $C A E^{13}$.

\section{Aquisição da agricultura familiar}

Foi observado que $81 \%(n=13)$ dos municípios adquiriram alimentos provenientes de produtores de âmbito local/regional. Foi observado também que $44 \%(n=7)$ dos respondentes afirmaram ter adquirido alimentos de outras regiões (distantes do município) para $\mathrm{AE}$, sob a justificativa de não haver produtor ou o produto desejado em âmbito local.

Alguns estudos relacionam a aquisição da $\mathrm{AF}$ e de produtores locais às características de produção agrícola da região e ao porte do município ${ }^{5,29}$. Segundo esses, regiões onde a produção é direcionada à agroindústria, há dificuldade da AF atender o volume necessário requerido pelo PNAE. Além disso, municípios de grande porte teriam dificuldades de suprir a demanda de alimentos com a quantidade ofertada pela $A F^{5,29}$. Porém, ao analisar a aquisição dos municípios deste estudo, percebe-se que a aquisição não teve relação com o porte. Ao avaliar o percentual de aquisição da AF desses municípios em 2014, foi observado que municípios de grande porte utilizaram de $6,28 \%$ a $69,91 \%$ dos recursos destinados pelo $\mathrm{FNDE}^{30}$. Isso pode indicar que a aquisição da AF em âmbito local possivelmente está relacionada “[...] à existência ou não de políticas municipais que incentivem a aproximação dos produtores locais ao mercado institucional de compra de alimentos vinculado ao PNAE [...] $]^{31 "}$.

Quanto à variedade de alimentos adquiridos da $A F$, o número de gêneros distintos adquiridos variou de 2 a 65 itens. As frutas provenientes da AF foram adquiridas por $88 \%(n=14)$ dos municípios, enquanto o grupo das carnes e ovos por apenas $25 \%(n=4)$. Estudos sobre a aquisição de alimentos da AF para o PNAE demonstram que os alimentos mais demandados são frutas e verduras ${ }^{32,33}$. A aquisição de carnes e ovos em menor quantidade pode estar relacionada aos padrões de qualidade exigidos em normativa, principalmente relacionados às legislações sanitárias e aos critérios de comprovação, como os "registros do produto e do estabelecimento nas respectivas instâncias responsáveis (dependendo do produto)"21,34:940. A legislação sanitária atual privilegia as grandes escalas de produção e dificulta a inclusão de produtos beneficiados da AF. Neste sentido, sugere-se a revisão e adequação da legislação sanitária à produção de pequena escala, para maior inclusão dos produtos da AF no mercado institucional $21,34,35$.

Ao analisar a forma de distribuição dos gêneros alimentícios, foi observado que $56 \%$ ( $n=9$ ) dos municípios afirmaram que os gêneros alimentícios da AF são entregues ponto a ponto (entrega em cada uma das escolas) e 38\% ( $n=6$ ) afirmaram que a entrega é centralizada (entrega dos gêneros alimentícios no depósito central). Um município de pequeno porte relatou que a SME busca os gêneros alimentícios na propriedade rural. Em relação ao porte dos municípios, é sabido que os de grande porte exigem maior quantidade de alimentos e possuem maior complexidade no planejamento para transporte, armazenamento e distribuição ${ }^{21,32}$. Porém, a exemplo do município de pequeno porte anteriormente citado, a gestão que apoia o produtor em relação à logística pode potencializar a inclusão da AF no Programa ${ }^{21,29}$. Além disso, há outras possibilidades de apoio, como: o agricultor poder escolher uma região da cidade na qual serão feitas as entregas; ou ainda escolher diretamente as escolas para as quais têm possibilidade de entregar; definir na chamada pública a periodicidade conveniente de entrega ${ }^{29}$. 
O desempenho predominantemente "regular" dos municípios avaliados aponta para fragilidades da gestão em relação ao desconhecimento e não aplicabilidade dos critérios previstos em normativa, especialmente quanto ao Planejamento de Cardápios, Chamada Pública e seleção dos Projetos de Vendas.

$\mathrm{Na}$ amostra selecionada, a avaliação da gestão do PNAE em relação ao fomento e aquisição da AF foi predominantemente "regular" e não apresentou diferença entre os portes dos municípios.

Quanto ao modelo de compras públicas estabelecido pela Lei no $11.947 / 2009^{6}$, é válido destacar que está em fase de aprimoramento e que, apesar das dificuldades, as aquisições de alimentos provenientes da agricultura familiar têm aumentado.

Para superação das dificuldades apontadas, faz-se necessário a articulação da gestão municipal com os agricultores para realização do mapeamento da vocação agrícola, bem como para organização da logística de entrega; responsabilização da gestão pela promoção de atividades de formação permanente dos atores envolvidos no PNAE; e do acesso da AF à assistência técnica.

\section{CONSIDERAÇÕES FINAIS}

Destaca-se que pesquisas de avaliação normativa possuem o potencial de evidenciar as prioridades de ação para a gestão. No âmbito municipal, a gestão do PNAE com enfoque ao fomento e aquisição de alimentos da AF deve ser fortalecida quanto aos indicadores avaliados neste estudo: Planejamento de Cardápio, Chamada Pública e Projetos de Venda.

Desta forma, faz-se premente a qualificação da gestão municipal do PNAE envolvendo secretários, nutricionistas, técnicos da administração e setor de compras, CAE, para adequada execução de todas as etapas da compra de alimentos da AF, tais como o planejamento de cardápios alinhados à produção local, tempo adequado e ampla divulgação da chamada pública, além de clareza quanto aos critérios de priorização dos produtores locais sobre os demais grupos. Por outro lado, reitera-se a importância da ATER para oportunizar a capacitação dos agricultores familiares na comercialização de alimentos aos mercados institucionais.

Ainda assim, algumas limitações podem estar associadas à metodologia quantitativa, já que são apontadas as lacunas em relação ao cumprimento das normativas, mas não exploram os motivos pelos quais a gestão não cumpre sua função institucional.

Neste sentido, o presente estudo oportuniza a construção de hipóteses para novas pesquisas com abordagens complementares que qualifiquem os achados deste artigo, tais como: estudos qualitativos de avaliação de impacto do PNAE; metodologias de estudo de caso; avaliação das repercussões do enfraquecimento das políticas de ATER e inclusão da AF nas compras institucionais e avaliação da influência dos mercados institucionais no desenvolvimento da AF.

\section{AGRADECIMENTOS}

Ao Fundo Nacional de Desenvolvimento da Educação (FNDE) pelo financiamento desta pesquisa.

\section{REFERÊNCIAS}

1. Triches RM. Repensando o mercado da alimentação escolar: novas institucionalidades para o desenvolvimento rural. In: Grisa C, Schneider S, orgs. Políticas públicas de desenvolvimento rural no Brasil. Porto Alegre: UFRGS; 2015. p. 181-200.

2. Brasil. Fundo Nacional de Desenvolvimento da Educação - FNDE. Programa Nacional de Alimentação Escolar - PNAE. Histórico [Internet]. Brasília: Ministério da Educação; 2019 [citado em 2019 Jun 20] Disponível em: https://www.fnde.gov.br/programas/pnae/pnae-sobre-o-programa/pnae-historico

3. Cazella AA, Capellesso AJ, Medeiros M, Tecchio A, Sencébé Y, Búrigo FL. Políticas públicas de desenvolvimento rural no Brasil: o dilema entre inclusão produtiva e assistência social. Polit Soc. 2016;15:49-79. http://dx.doi.org/10.5007/2175-7984.2016v15nesp1p49. 
4. Peixinho AML. A trajetória do Programa Nacional de Alimentação Escolar no período de 2003-2010: relato do gestor nacional. Cien Saude Colet. 2013;18(4):909-16. http://dx.doi.org/10.1590/S141381232013000400002. PMid:23670366.

5. Machado PMO. Características do processo de aquisição de alimentos da agricultura familiar pelo Programa Nacional de Alimentação Escolar: um estudo transversal exploratório do universo de municípios brasileiros. [Dissertação]. Florianópolis: Universidade Federal de Santa Catarina; 2013.

6. Brasil. Lei no 11.947, de 16 de junho de 2009. Dispõe sobre o atendimento da alimentação escolar e do Programa Dinheiro Direto na Escola aos alunos da educação básica; altera as Leis nos 10.880, de 9 de junho de 2004, 11.273, de 6 de fevereiro de 2006, 11.507, de 20 de julho de 2007; revoga dispositivos da Medida Provisória no 2.178-36, de 24 de agosto de 2001, e a Lei no 8.913, de 12 de julho de 1994; e dá outras providências. Diário Oficial da União, Brasília,17 de junho de 2009.

7. Contandriopoulos A, Champagne F, Denis JL, Pineault R. A avaliação na área da saúde: conceitos e métodos. In: Hartz ZMA, organizador. Avaliação em Saúde: dos modelos conceituais à prática na análise da implantação de programas. Rio de Janeiro: Fiocruz; 1997. p. 29-48.

8. Jannuzzi PM. Monitoramento e avaliação de programas sociais: uma introdução aos conceitos e técnicas. Campinas: Alínea; 2016.

9. Instituto Brasileiro de Geografia e Estatística. Síntese das Informações Municipais. IBGE Cidades@ [Internet]. Rio de Janeiro: IBGE; 2015 [citado em 2018 Jul 20]. Disponível em: http://www.cidades.ibge.gov.br/xtras/ home.php

10. Instituto Nacional de Estudos e Pesquisas Educacionais Anísio Teixeira - INEP. Avaliação do Programa Nacional de Alimentação Escolar (PNAE): censo escolar 2004 [Internet]. Brasília: INEP; 2007 [citado em 2016 Dez 20]. Disponível em: http://download.inep.gov.br/download/estudos_pesquisas/financ_gasto/ pnae_relatorio20 04_final.pdf

11. Gabriel CG. Programa Nacional de Alimentação Escolar: Construção de modelo de avaliação da gestão municipal. [tese]. Florianópolis: Programa de Pós-graduação em Saúde Coletiva, Universidade Federal de Santa Catarina; 2013.

12. Brasil. Ministério da Educação. Fundo Nacional de Desenvolvimento da Educação - FNDE. Resolução nº 26, de 17 de junho de 2013. Dispõe sobre o atendimento da alimentação escolar aos alunos da educação básica no âmbito do Programa Nacional de Alimentação Escolar - PNAE. Diário Oficial da União [Internet], Brasília, 2013 [citado em 16 nov 2016]. Disponível em: https://www.fnde.gov.br/fndelegis/action/ UrlPublicasAction.php

13. Brasil. Ministério da Educação. Fundo Nacional de Desenvolvimento da Educação - FNDE. Resolução CD/ FNDE n 04 de 03 de abril de 2015. Altera a redação dos artigos 25 a 32 da Resolução/CD/FNDE n²6, de 17 de junho de 2013, no âmbito do Programa Nacional de Alimentação Escolar (PNAE). Diário Oficial da União [Internet], Brasília, 2013 [citado em 21 Nov 2016]. Disponível em: http://www.fnde.gov.br/fnde/ legislacao/resolucoes/item/6341- resolu\%C3\%A7\%C3\%A3o-cd-fnde-mec-n\%C2\%BA-4,-de-3-de-abrilde-2015

14. Brasil. Lei $\mathrm{n}^{\circ} 11.346$ de 15 de setembro de 2006. Cria o Sistema Nacional de Segurança Alimentar e Nutricional - SISAN com vistas em assegurar o direito humano à alimentação adequada e dá outras providências. Diário Oficial da União [Internet], Brasília, 2006. [citado em 21 Nov 2016]. Disponível em: https://www.planalto.gov.br/ccivil_03/_ato2004-2006/2006/lei//11346.htm

15. Empresa de Pesquisa Agropecuária e Extensão Rural de Santa Catarina - EPAGRI. Institucional [Internet]. [citado em 30 Dez 2016]. Disponível em: http://www.epagri.sc.gov.br/?page_id=5767

16. Brasil. Fundo Nacional de Desenvolvimento da Educação - FNDE. Programa Nacional de Alimentação Escolar - PNAE. Centros Colaboradores e UAE [Internet]. 2012 [citado em 30 Dez 2016]. Disponível em: http://www.fnde.gov.br/programas/alimentacao-escolar/centroscolaboradores-e-uae/o-que-sao

17. Belik W. A heterogeneidade e suas implicações para as políticas públicas no rural brasileiro. Rev Econ Sociol Rural. 2015;53(1):9-30. http://dx.doi.org/10.1590/1234-56781806-9479005301001.

18. Gabriel CG, Goulart G, Calvo MCM. Gestão municipal do Programa Nacional de Alimentação Escolar nas capitais da região Sul do Brasil. Rev Nutr. 2015;28(6):667-80. http://dx.doi.org/10.1590/141552732015000600009.

19. Mossmann MP, Teo CRPA, Busato MA, Triches RM. Interface between family farming and school feeding: barriers and coping mechanisms from the perspective of different social actors in Southern Brazil. Rev Econ Sociol Rural. 2017;55(2):325-42. https://doi.org/10.1590/1234-56781806-94790550207.

20. Brasil. Conselho Nacional de Segurança Alimentar e Nutricional. Construção do Sistema e da Política Nacional de Segurança Alimentar e Nutricional: a experiência brasileira [Internet]. Rome: FAO; 2009 [citado em 30 dez 2016]. Disponível em: https://www.fao.org.br/download/Seguranca_Alimentar_Portugues.pdf 
21. Sousa AA, Silva APF, Azevedo E, Ramos MO. Cardápios e sustentabilidade: ensaio sobre as diretrizes do Programa Nacional de Alimentação Escolar. Rev Nutr. 2015;28(2):217-29. http://dx.doi.org/10.1590/141552732015000200010.

22. Brasil. Conselho Federal de Nutricionistas. Resolução $n^{\circ} 465$ de 17 de julho de 2010. Dispõe sobre as atribuições do Nutricionista, estabelece parâmetros numéricos mínimos de referência no âmbito do Programa de Alimentação Escolar (PAE) e dá outras providências. Diário Oficial da União [Internet], Brasília, 2010 [citado em 21 Nov 2016]. Disponível em: http://www.cfn.org.br/novosite/arquivos/Resol-CFN-465atribuicao-nutricionista-PAE.pdf

23. Silva APF, Sousa AA. Alimentos orgânicos da agricultura familiar no Programa Nacional de alimentação Escolar do Estado de Santa Catarina, Brasil. Rev Nutr. 2013;26(6):701-14. http://dx.doi.org/10.1590/S141552732013000600009.

24. Chaves LG, SantanaTC, Gabriel CG, Vasconcelos FA. Reflexões sobre a atuação do nutricionista no Programa Nacional de Alimentação Escolar no Brasil. Cien Saude Colet. 2013;18(4):917-26. http://dx.doi.org/10.1590/ S1413-81232013000400003. PMid:23670367.

25. Melo MNT, Sá RMPF, Melo DA Fo. Sustentabilidade de um programa de alimentação escolar bemsucedido: estudo de caso no Nordeste do Brasil. Cien Saude Colet. 2016;21(6):1899-908. http://dx.doi. org/10.1590/1413-81232015216.08752016. PMid:27276541.

26. Soares P, Martinelli SS, Melgarejo L, Davó-Blanes MC, Cavalli SB. Potencialidades e dificuldades para o abastecimento da alimentação escolar mediante a aquisição de alimentos da agricultura familiar em um município brasileiro. Cien Saude Colet. 2015 jun;20(6):1891-900. http://dx.doi.org/10.1590/141381232015206.16972014. PMid:26060967.

27. Saraiva EB, Silva APF, Sousa AA, Cequeira GF, Chagas CMS, Toral N. Panorama da compra de alimentos da agricultura familiar para o Programa Nacional de Alimentação Escolar. Cien Saude Colet. 2013;18(4):92735. http://dx.doi.org/10.1590/S1413-81232013000400004. PMid:23670368.

28. Centro Colaborador em Alimentação e Nutrição do Escolar de Santa Catarina - CECANE-SC. [Internet]. 2016 [citado em 30 Dez 2016]. Disponível em: http://cecanesc.ufsc.br/sitio/sitio/index/idsitio/1\#/

29. Brasil. Ministério do Desenvolvimento Agrário. Secretaria da Agricultura Familiar. Instituto Via Pública. Projeto Nutre SP: análise da inclusão da agricultura familiar na alimentação escolar no estado de São Paulo. In: Corá MAJ, Belik W, organizadores. A Agricultura Familiar e o Atendimento à Demanda Institucional das Grandes Cidades. São Paulo: Instituto Via Pública; 2012 [citado em 26 Dez 2016]. Disponível em: https:// www.google.com.br/url?sa=t\&rct=j\&q=\&esrc=s\&source=web\&cd=1\&ved=0ahUK EwjY4aiKm7bRAhXG MSYKHfOLBJEQFggcMAA\&url=http\%3A\%2F\%2Fportal.mda.gov. br\%2Fo\%2F9793815\&usg=AFQjCNFc 9HUmEDAzRvfiPgullB4k02MMBQ\&sig2=_4d5iT7 y25dPLqZPEwhxyQ

30. Belik W, Chaim NA. O programa Nacional de Alimentação Escolar e a gestão municipal: eficiência administrativa, controle social e desenvolvimento local. Rev Nutr. 2009;22(5):595-607. http://dx.doi. org/10.1590/S1415-52732009000500001.

31. Brasil. Fundo Nacional de Desenvolvimento da Educação - FNDE. Programa Nacional de Alimentação Escolar - PNAE. Dados da Agricultura Familiar [Internet]. Brasília: FNDE; 2016 [citado em 27 Dez 2016]. Disponível em: http://www.fnde.gov.br/programas/alimentacao-escolar/alimentacao-escolarconsultas/ dados-da-agricultura-familiar

32. Amorim ALB, Rosso VV, Bandoni DH. Acquisition of family farm foods for school meals: Analysis of public procurements within rural family farming published by the cities of São Paulo state. Rev Nutr. 2016;29(2):297-306. http://dx.doi.org/10.1590/1678-98652016000200012.

33. Vidal GM, Veiros MB, Sousa AA. School menus in Santa Catarina: Evaluation with respect to the National School Food Program regulations. Rev Nutr. 2015;28(3):277-87. http://dx.doi.org/10.1590/141552732015000300005.

34. Triches RM, Schneider S. Alimentação escolar e agricultura familiar: reconectando o consumo à produção. Saude Soc. 2010;19(4):933-45. http://dx.doi.org/10.1590/S0104-12902010000400019.

35. Triches RM, BelikW. Alimentação escolar: construindo interfaces entre saúde, educação e desenvolvimento. In:Teo CRPA, Triches RM, organizadores. Alimentação escolar: construindo interfaces entre saúde, educação e desenvolvimento. Chapecó: Argos; 2016. p. 84. 